\title{
First record of Pachybrachis Chevrolat, 1836 (Coleoptera: Chrysomelidae: Cryptocephalinae) on Spermacoce verticillata L. (Rubiaceae) and synthesis of its plant associations in Brazil
}

\author{
Primer registro de Pachybrachis Chevrolat, 1836 (Coleoptera: Chrysomelidae: Cryp- \\ tocephalinae) en Spermacoce verticillata L. (Rubiaceae) y síntesis de sus asociaciones \\ vegetales en Brasil
}

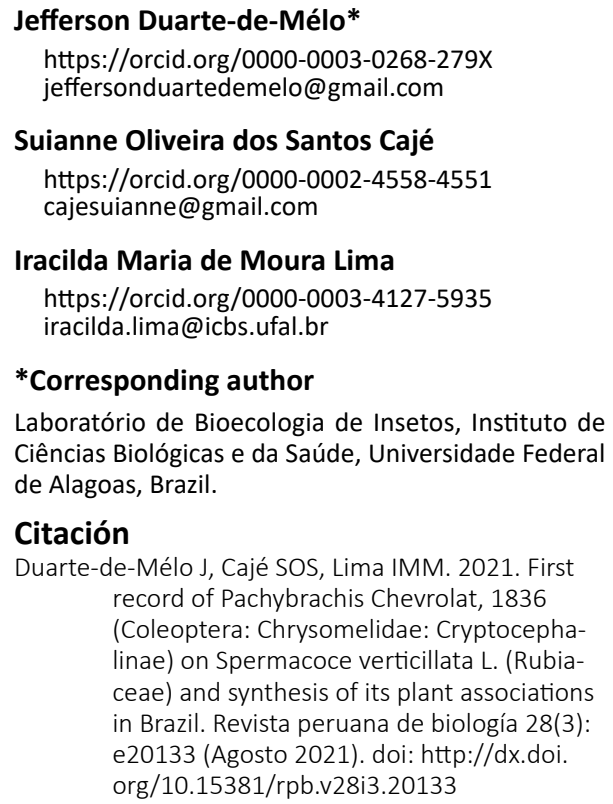

Suianne Oliveira dos Santos Cajé

https://orcid.org/0000-0002-4558-4551 cajesuianne@gmail.com

Iracilda Maria de Moura Lima

https://orcid.org/0000-0003-4127-5935

iracilda.lima@icbs.ufal.br

*Corresponding author

Laboratório de Bioecologia de Insetos, Instituto de Ciências Biológicas e da Saúde, Universidade Federal de Alagoas, Brazil.

\section{Citación}

Duarte-de-Mélo J, Cajé SOS, Lima IMM. 2021. First record of Pachybrachis Chevrolat, 1836 (Coleoptera: Chrysomelidae: Cryptocephalinae) on Spermacoce verticillata L. (Rubiaceae) and synthesis of its plant associations in Brazil. Revista peruana de biología 28(3): e20133 (Agosto 2021). doi: http://dx.doi. org/10.15381/rpb.v28i3.20133

Abstract

This study reports Pachybrachis sp. association with a new host, Spermacoce verticillata L. (Rubiaceae). It synthesizes Brazil locality records and host plant records from literature. Specimens of adults and larvae were collected on the host in an urban area of Maceió, Alagoas, Brazil. Larvae and adults were reared on the host plant in the laboratory, using fresh plant tissue daily.

\section{Resumen}

Este estudio presenta la asociación de Pachybrachis sp. con un nuevo huésped, Spermacoce verticillata $\mathrm{L}$. (Rubiaceae). En este trabajo se dan a conocer las localidades registradas en Brasil para la especie y los registros de plantas huésped mencionados en la literatura. Se recolectaron especímenes de adultos y larvas sobre el huésped en un área urbana de Maceió, Alagoas, Brasil. Las larvas y los adultos se criaron en la planta huésped en el laboratorio, utilizando tejido vegetal fresco a diario.

\section{Keywords:}

Alagoas; Host plant; Immature; Insect-plant interaction; Pachybrachina.

Palabras clave

Alagoas; Inmaduro; Interacción insecto-planta; Pachybrachina; Planta huésped.

Spermacoce verticillata L. (synonym: Borreria verticillata (L.) G. Mey.) (Rubiaceae) conserves important pollinators and natural enemies around it (Dale et al. 2019). Commonly known as "shrubby false buttonweed" (GBIF 2021a), S. verticillata is a native herb from tropical America, but it is considered a weed in Brazil probably due to its tolerance to nutrient-poor soils and the easy dispersal of its seeds (Lorenzi 1991). This plant is part of folk medicine due to its antimicrobial (Koné et al. 2004) and anti-inflammatory properties (Lima et al. 2018); it is also a source of raw material for handicraft products (Lima et al. 2013). Spermacoce verticillata is also useful in biological control programs as it is the main source of nectar for wasps (Arévalo \& Frank 2005, Leppla et al. 2007, Abraham et al. 2010, Portman et al. 2010). In addition, it has larvicidal activity against Aedes aegypti (Linnaeus, 1762) (Oliveira et al. 2010). 
Chrysomelidae is the fourth largest family of the order Coleoptera. Because it is essentially phytophagous, it plays a big role in insect-plant interactions. Members of the subfamily Cryptocephalinae, represented in Brazil by 723 species in 37 genera (Chamorro et al. 2021), feed on green leaves, decaying plant material, and girdle stems (Chamorro 2014); some are myrmecophilous (Agrain et al. 2015). Members of the Cryptocephalini tribe are flower eaters and pollen eaters (Chamorro 2014). Some groups, as subtribe Pachybrachina, pose a complex identification challenge at genus level even for specialists (Sassi 2018).

Worldwide, two species are associated with Rubiaceae: Cryptocephalus trizonatus Suffrian, 1852 on Coffea sp. (Maes \& Staines 1991) and Cryptocephalus moraei (Linnaeus, 1758) on Gallium sp. (Biondi \& Di Casoli 1996, Maican \& Munteanu 2008). None of the reports provide information about the presence of immatures on the plant.

We report a new record of Pachybrachis sp. whose adults and immature stages are in association with $S$. verticillata in Brazil. We also summarize the distribution records of this genus and its plant associations in Brazilian territory.
Two adults and two mature larvae specimens were collected in an urban area of Maceió, Alagoas (-9.558304, -35.776127) (Fig. 1), on S. verticillata (Fig. 2), near the Catolé e Fernão Velho Environmental Protection Area, fragment of the Atlantic Forest, in September of 2019. The plant was identified by Dra. Letícia Ribes de Lima (Universidade Federal de Alagoas). In the laboratory $\left(24.1-26.0{ }^{\circ} \mathrm{C}, 62-77 \% \mathrm{RH}\right.$, photoperiod $12: 12 \mathrm{~h}$ ), the larvae and adults were reared with daily addition of fresh parts of the plant, from which pupae and eggs were obtained. Morphometric determinations under stereomicroscope were performed too. Subsequently, a genera key (Chamorro-Lacayo 2013) of the subtribe Pachybrachina was used for identification of the beetles, which was confirmed by Dr. Davide Sassi (Università degli Studi di Milano). The analysis of publications related to the occurrence records made it possible to trace the current state of the genus occurrence and plant association for Brazil. Voucher specimens of the beetles will be deposited in the Padre Jesus Santiago Moure Entomological Collection, Federal University of Paraná, Brazil; plant exsiccates will be deposited in the Institute of the Environment of Alagoas's Herbarium-MAC.
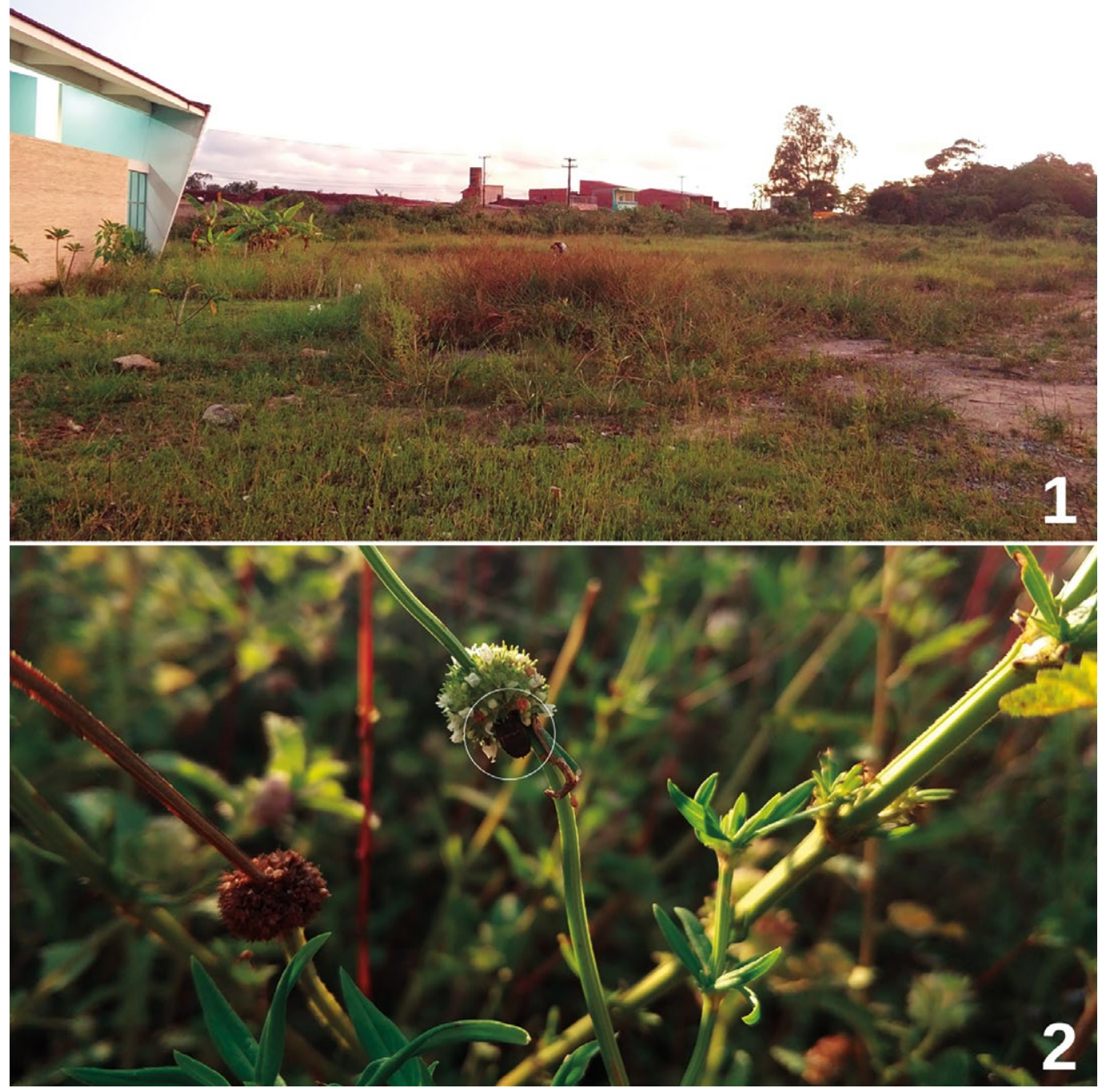

Figures 1-2. Habitat of Pachybrachis sp. 1: Location where the specimens were found. 2: Host plant Spermacoce verticillata with a late instar larva of Pachybrachis sp. feeding on its inflorescence (white circle). 
The beetles were identified as Pachybrachis Chevrolat, 1836 (Chrysomelidae: Cryptocephalinae: Cryptocephalini) (Figs. 3, 4), with female, $\approx 3.2 \mathrm{~mm}$ long $(\mathrm{n}=$ 1 ), being larger and more robust than the male, $\approx 3.0$ $\mathrm{mm}$ long $(\mathrm{n}=1)$. The following characters were confirmed by the key to identify specimens as in the genus Pachybrachis and differentiating it from similar genera such as Metallactus Suffrian, 1866 and Griburius Haldeman, 1849: (a) intercoxal process of the proesternum do not go further the posterior margin of the protrax; (b) broad and evident punctuations in the head, prothorax and elytra; (c) baseline of the elytra is wider than prothorax posterior line; and (d) dorsally flat prothorax, not domed. The egg fecal capsule is $\approx 0.7 \mathrm{~mm}$ long ( $\mathrm{n}=6$ ) (Fig. 5) and the pupa fecal case is $\approx 5.1 \mathrm{~mm}$ long $(n=2)$ (Fig. 6).
Pachybrachis sp. adults feed on leaves of $S$. verticilla$t a$, while late instar larvae feed on inflorescences. Mature larvae seem to seek a position under the inflorescences, where they feed until pupation. Females lay their eggs on the inflorescences, but eggs were also found on substrate.

The genus Pachybrachis (220 species) is poorly studied in Brazil, with scarce and incomplete records. There is not a single record registered for Brazil in the GBIF (GBIF 2021b), the largest biodiversity data network. Ten studies have information about the genus Pachybrachis in Brazil (Table 1). The characteristics of the exact locations of occurrence are barely presented. Most (Baly 1877, Bowditch 1913, Albertoni 2008, Dias 2016, Guedes et al. 2019, Rafael et al. 2020) do not provide any information about plants. Only one study reports the presence and collection of immatures.

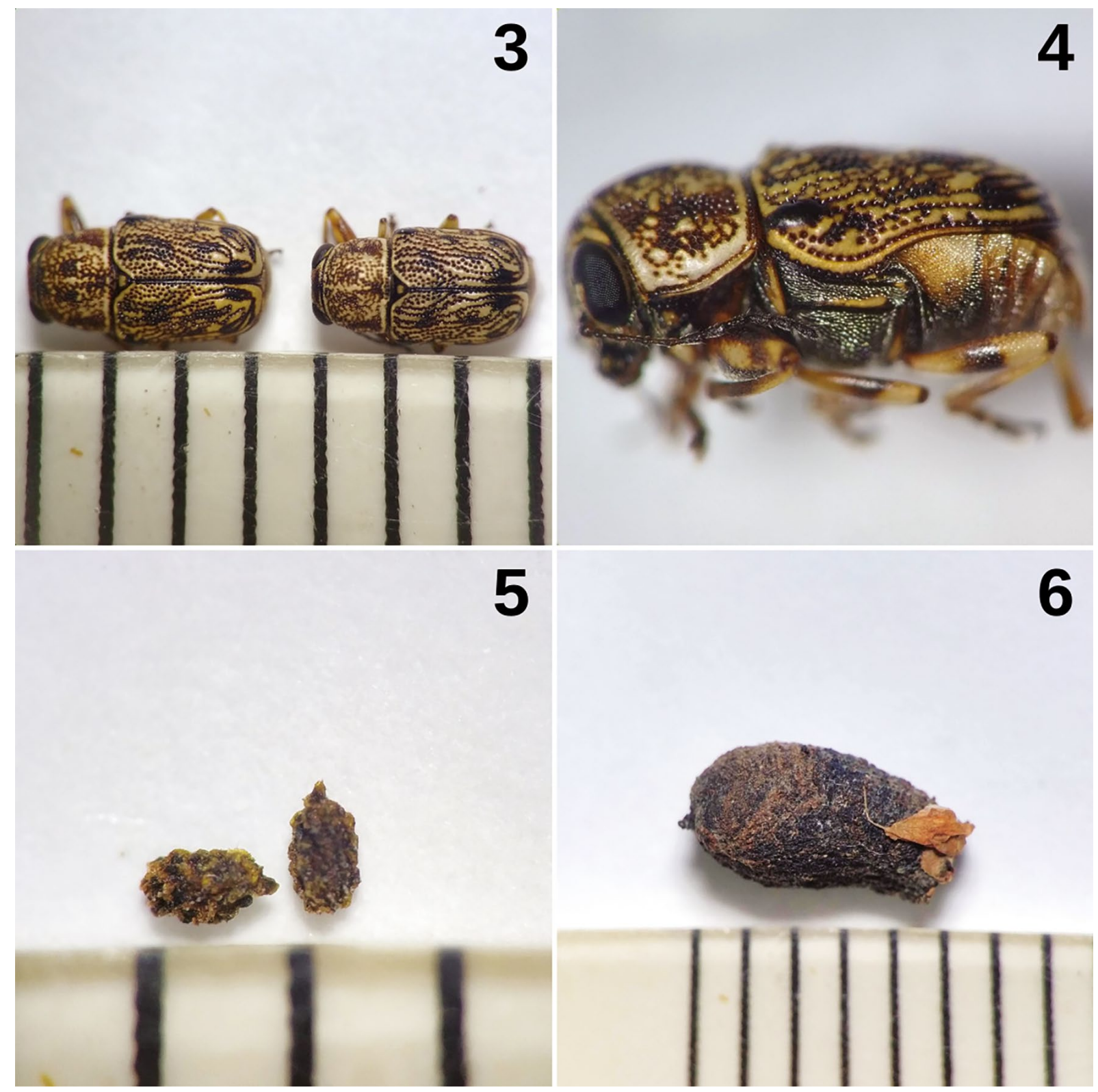

Figures 3-6. Adults and immatures of Pachybrachis sp. from northeastern Brazil. 3: Female (left) and male (right) in dorsal view. 4: Female in lateral view. 5: Egg fecal capsules. 6: Pupa fecal case. 
Four records provide information about the plants on which the specimens were found. The genus was recorded on Mimosa pigra L. (Fabaceae) and on Mimosa pigra var. berlandieri (A.Gray) B.L.Turner (accepted name: $M i$ mosa pigra var. asperata (L.) Zarucchi, Vincent \& Gandhi) (Fabaceae) (Harley et al. 1995); on Baccharis stylosa Gardner (Asteraceae) in a high altitude area (above 1600 meters) (Flinte et al. 2009); on Ipomoea carnea subsp. fistulosa (Mart. ex Choisy) D.F.Austin (Convolvulaceae) in
Caatinga (Martins 2015); and on Chamaecrista cathartica (Mart.) H.S.Irwin \& Barneby (Fabaceae) in urban area (Lopes 2020). However, only B. stylosa was confirmed as a host plant as immatures and adults were feeding on it.

In summary, we present as a novelty Pachybrachis sp. on $S$. verticillata, the first record of Rubiaceae as a host for this beetle genus. Not least, this is the first report of Pachybrachis immatures from Brazil.

Table 1. Occurrences of Pachybrachis Chevrolat, 1836 and its plant associations in Brazil.

\begin{tabular}{|c|c|c|c|c|c|c|c|}
\hline $\begin{array}{l}\text { Pachybrachis (name as } \\
\text { reported) }\end{array}$ & State & $\begin{array}{l}\text { Municipality or } \\
\text { Distrite }\end{array}$ & Location & $\begin{array}{l}\text { Phytophy- } \\
\text { siognomy }\end{array}$ & $\begin{array}{l}\text { Plant (name } \\
\text { as reported) }\end{array}$ & $\begin{array}{l}\text { Notes on plant } \\
\text { association }\end{array}$ & Literature \\
\hline Pachybrachys contortus & Pará & Santarém & $\begin{array}{l}\text { Banks of the } \\
\text { Amazon }\end{array}$ & - & - & - & Baly 1877 \\
\hline Pachybrachys contortus & Ceará & - & - & - & - & - & Bowditch 1913 \\
\hline Pachybrachys contortus & Ceará & Maranguape & - & - & - & - & Bowditch 1913 \\
\hline Pachybrachys sp. 1 & Ceará & Independência & - & - & - & - & Bowditch 1913 \\
\hline Pachybrachys sp. 2 & Ceará & Independência & - & - & - & - & Bowditch 1913 \\
\hline Pachybrachys sp. 3 & Ceará & Independência & - & - & - & - & Bowditch 1913 \\
\hline Pachybrachys sp. 4 & $\begin{array}{l}\text { Rio Grande do } \\
\text { Norte }\end{array}$ & Natal & - & - & - & - & Bowditch 1913 \\
\hline Pachybrachis contortus & Minas Gerais & - & - & - & Mimosa pigra & $\begin{array}{l}\text { The authors just } \\
\text { report that adults } \\
\text { were found on } \\
\text { plant. Nothing } \\
\text { about feeding. }\end{array}$ & Harley et al. 1995 \\
\hline $\begin{array}{l}\text { Pachybrachis sp. nr. } \\
\text { musiva }\end{array}$ & Minas Gerais & - & - & - & Mimosa pigra & $\begin{array}{l}\text { The authors just } \\
\text { report that adults } \\
\text { were found on } \\
\text { plant. Nothing } \\
\text { about feeding. }\end{array}$ & Harley et al. 1995 \\
\hline Pachybrachis sp. & $\begin{array}{l}\text { Amazonas, Minas } \\
\text { Gerais, Pará, Pa- } \\
\text { raná, São Paulo }\end{array}$ & - & - & - & Mimosa pigra & $\begin{array}{l}\text { The authors just } \\
\text { report that adults } \\
\text { were found on } \\
\text { plant. Nothing } \\
\text { about feeding. }\end{array}$ & Harley et al. 1995 \\
\hline Pachybrachis sp. & $\begin{array}{l}\text { Amazonas, Minas } \\
\text { Gerais, Pará, Pa- } \\
\text { raná, São Paulo }\end{array}$ & - & - & - & $\begin{array}{l}\text { Mimosa pigra } \\
\text { var. berlandieri }\end{array}$ & $\begin{array}{l}\text { The authors just } \\
\text { report that adults } \\
\text { were found on } \\
\text { plant. Nothing } \\
\text { about feeding. }\end{array}$ & Harley et al. 1995 \\
\hline Pachybrachis sp. 1 & Santa Catarina & - & - & - & - & - & Albertoni 2008 \\
\hline Pachybrachis sp. 2 & Santa Catarina & - & - & - & - & - & Albertoni 2008 \\
\hline Pachybrachis sp. & Rio de Janeiro & - & $\begin{array}{l}\text { Serra dos Orgãos } \\
\text { National Park }\end{array}$ & $\begin{array}{l}\text { High-montane } \\
\text { forest }\end{array}$ & $\begin{array}{l}\text { Baccharis } \\
\text { stylosa }\end{array}$ & $\begin{array}{l}\text { Plant should be } \\
\text { considered a host } \\
\text { plant because } \\
\text { adults and larvae } \\
\text { were found fee- } \\
\text { ding on plant. }\end{array}$ & Flinte et al. 2009 \\
\hline Pachybrachis sp. & Rio de Janeiro & - & $\begin{array}{l}\text { Serra dos Orgãos } \\
\text { National Park }\end{array}$ & $\begin{array}{l}\text { High altitude } \\
\text { grassland }\end{array}$ & $\begin{array}{l}\text { Baccharis } \\
\text { stylosa }\end{array}$ & $\begin{array}{l}\text { Plant should be } \\
\text { considered a host } \\
\text { plant because } \\
\text { adults and larvae } \\
\text { were found fee- } \\
\text { ding on plant. }\end{array}$ & Flinte et al. 2009 \\
\hline Pachybrachis sp. & Paraíba & - & Caatinga & - & $\begin{array}{l}\text { Ipomoea } \\
\text { carnea ssp. } \\
\text { fistulosa }\end{array}$ & $\begin{array}{l}\text { Plant could be } \\
\text { considered a food } \\
\text { plant because } \\
\text { adult was found } \\
\text { feeding on plant. }\end{array}$ & Martins 2015 \\
\hline Pachybrachys sp. & Rio Grande do Sul & Aceguá & Pampa & - & - & - & Dias 2016 \\
\hline Pachybrachys sp. 1 & Paraíba & Santa Terezinha & Caatinga & $\begin{array}{l}\text { Riparian } \\
\text { forest }\end{array}$ & - & - & $\begin{array}{l}\text { Guedes et al. } \\
2019\end{array}$ \\
\hline
\end{tabular}


Table 1. ...

\begin{tabular}{|c|c|c|c|c|c|c|c|}
\hline $\begin{array}{l}\text { Pachybrachis (name as } \\
\text { reported) }\end{array}$ & State & $\begin{array}{l}\text { Municipality or } \\
\text { Distrite }\end{array}$ & Location & $\begin{array}{l}\text { Phytophy- } \\
\text { siognomy }\end{array}$ & $\begin{array}{l}\text { Plant (name } \\
\text { as reported) }\end{array}$ & $\begin{array}{l}\text { Notes on plant } \\
\text { association }\end{array}$ & Literature \\
\hline Pachybrachys sp. 2 & Paraíba & Santa Terezinha & Caatinga & $\begin{array}{l}\text { Riparian } \\
\text { forest and } \\
\text { xerophilous } \\
\text { vegetation }\end{array}$ & - & - & $\begin{array}{l}\text { Guedes et al. } \\
2019\end{array}$ \\
\hline Pachybrachys sp. 3 & Paraíba & Santa Terezinha & Caatinga & $\begin{array}{l}\text { Riparian } \\
\text { forest and } \\
\text { xerophilous } \\
\text { vegetation }\end{array}$ & - & - & $\begin{array}{l}\text { Guedes et al. } \\
2019\end{array}$ \\
\hline Pachybrachys sp. 4 & Paraíba & Santa Terezinha & Caatinga & $\begin{array}{l}\text { Riparian } \\
\text { forest }\end{array}$ & - & - & $\begin{array}{l}\text { Guedes et al. } \\
2019\end{array}$ \\
\hline Pachybrachis sp. & Minas Gerais & Uberlândia & Urban area & (Don't apply) & $\begin{array}{l}\text { Chamaecrista } \\
\text { cathartica }\end{array}$ & $\begin{array}{l}\text { Plant could be } \\
\text { considered a food } \\
\text { plant because } \\
\text { adult rearing on } \\
\text { plant was perfor- } \\
\text { med. }\end{array}$ & Lopes 2020 \\
\hline Pachybrachis sp. & Pernambuco & $\begin{array}{l}\text { Fernando de } \\
\text { Noronha }\end{array}$ & $\begin{array}{l}\text { Fernando de } \\
\text { Noronha Marine } \\
\text { National Park }\end{array}$ & - & - & - & Rafael et al. 2020 \\
\hline
\end{tabular}

\section{Literature cited}

Abraham CM, Held DW, Wheeler C. 2010. Seasonal and diurnal activity of Larra bicolor (Hymenoptera: Crabronidae) and potential ornamental plants as nectar sources. Applied Turfgrass Science 7(1):1-9. https://doi. org/10.1094/ATS-2010-0312-01-RS

Agrain FA, Buffington ML, Chaboo CS, Chamorro ML, Schöller M. 2015. Leaf beetles are ant-nest beetles: the curious life of the juvenile stages of case-bearers (Coleoptera, Chrysomelidae, Cryptocephalinae). In: Jolivet P, Santiago-Blay J, Schmitt M, editors. Research on Chrysomelidae 5. ZooKeys 547:133-164. https://doi. org/10.3897/zookeys.547.6098

Albertoni FF. 2008. Besouros da restinga do entorno da Lagoa Pequena, Florianópolis, SC: levantamento taxonômico e aspectos ecológicos [bachelor's monograph]. Florianópolis (SC): Universidade Federal de Santa Catarina.

Arévalo HA, Frank JH. 2005. Nectar sources for Larra bicolor (Hymenoptera: Sphecidae), a parasitoid of Scapteriscus mole crickets (Orthoptera: Gryllotalpidae), in northern Florida. Florida Entomologist 88(2):146-151. https://doi.org/10.1653/00154040(2005)088[0146:NSFLBH]2.0.CO;2

Baly JS. 1877. XVI. Descriptions of new genera and species of Cryptocephalidae. Transactions of the Royal Entomological Society of London 25(3):211-232. https:// biostor.org/reference/235678

Biondi M, Di Casoli MT. 1996. Il popolamento a Coleoptera Chrysomelidae del massiccio del Gran Sasso: analisi zoogeografica ed autoecologica (Insecta). In: Cicolani B, editor. Monitoraggio biologico del Gran Sasso. Volume 1. Colledara (TE): Andromeda Editrice. p. 176-198.

Bowditch FC. 1913. The Phytophaga (Except Cassidae and Hispidae) of the Stanford Expedition to Brazil. Psyche: A Journal of Entomology 20(4):125-131. https://doi. org/10.1155/1913/94781
Chamorro-Lacayo ML. 2013. On the identity of Mastacanthus Suffrian, 1852 and Sternoglosus Suffrian, 1866 and key to world genera of Pachybrachina (Chrysomelidae: Cryptocephalinae: Cryptocephalini). Caucasian Entomological Bulletin 9(1):201-206. https://doi. org/10.23885/1814-3326-2013-9-1-201-206

Chamorro ML. 2014. Cryptocephalinae Gyllenhal, 1813. In: Leschen RAB, Beutel RG, volume editors. Coleoptera, Beetles. Volume 3: Morphology and Systematics (Phytophaga). In: Kristensen NP, Beutel RG, editors. Handbook of Zoology Arthropoda: Insecta. Berlin/ Boston: De Gruyter. p. 230-236.

Chamorro ML, Sekerka L, Agrain F, Regalin R. 2021. Cryptocephalinae in Catálogo Taxonômico da Fauna do Brasil. PNUD. [accessed 2021 May 18] http://fauna.jbrj. gov.br/fauna/faunadobrasil/118309.

Dale AG, Perry RL, Cope GC, Benda N. 2019. Floral abundance and richness drive beneficial arthropod conservation and biological control on golf courses. Urban Ecosystems 23:55-66. https://doi.org/10.1007/s11252019-00907-0

Dias AC. 2016. Efeito de diferentes intensidades de pastejo sobre a comunidade de artrópodes nos campos sulinos [master's dissertation]. Porto Alegre (RS): Universidade Federal do Rio Grande do Sul.

Flinte V, Macedo MV, Monteiro RF. 2009. Chrysomelids and their host plants along an altitudinal gradient in an Atlantic rain forest in the State of Rio de Janeiro, Brazil. In: Jolivet P, Santiago-Blay J, Schmitt M, editors. Research on Chrysomelidae. Volume 2. Morphology and systematics (Phytophaga). Leiden: Brill. p. 31-56.

GBIF (Global Biodiversity Information Facility). 2021a. Spermacoce verticillata L. [accessed 2021 May 17] https:// www.gbif.org/species/2918574.

GBIF (Global Biodiversity Information Facility). 2021b. Pachybrachis Chevrolat, 1836. [accessed 2021 Mar 26] https://www.gbif.org/species/1047958. 
Guedes RS, Zanella FCV, Grossi PC. 2019. Composição e riqueza de espécies de uma comunidade de Coleoptera (Insecta) na Caatinga. Iheringia, Série Zoologia 109:e2019012. https://doi.org/10.1590/ $1678-4766 \mathrm{e} 2019012$

Harley K, Gillett J, Winder J, Forno W, Segura R, Miranda H, Kassulke R. 1995. Natural Enemies of Mimosa pigra and $\mathrm{M}$. berlandieri (Mimosaceae) and prospects for biological control of M. pigra. Environmental Entomology 24(6):1664-1678. https://doi.org/10.1093/ ee/24.6.1664

Leppla NC, Frank JH, Adjei MB, Vicente NE. 2007. Management of pest mole crickets in Florida and Puerto Rico with a nematode and parasitic wasp. Florida Entomologist 90(1):229-233. https://doi.org/10.1653/ 0015-4040(2007)90[229:MOPMCI]2.0.CO;2

Lima IC, Castro RN, Chaves DSA, Ferreira RT, Carvalho MF, Malvar DC, Vanderlinde FA. 2018. The folk medicine as tool for discovery of new anti-inflammatory drugs: the example of Spermacoce verticillata. Acta Horticulturae 1198:67-74. https://doi.org/10.17660/ActaHortic.2018.1198.13

Lima VHM, Gomes Neto JP, Cavalcanti MS. 2013. Recursos vegetais utilizados pela comunidade de Vila Velha, Ilha de Itamaracá, Pernambuco, Brasil. Revista Ouricuri 3(1):29-42. https://www.revistas.uneb.br/index. php/ouricuri/article/view/6431

Lopes BS. 2020. Ecologia das interações e diversidade de insetos em espécies de Fabaceae no Cerrado [doctoral's thesis]. Ribeirão Preto (SP): Universidade de São Paulo.

Lorenzi H. 1991. Plantas daninhas do Brasil: terrestres, aquáticas, parasitas, tóxicas e medicinais. $3^{\mathfrak{a}}$ ed. Nova Odessa (SP): Plantarum.

Koné W, Atindehou KK, Terreaux C, Hostettmann K, Traoré D, Dosso M. 2004. Traditional medicine in North Côted'Ivoire: screening of 50 medicinal plants for antibacterial activity. Journal of Ethnopharmacology 93(1):4349. https://doi.org/10.1016/j.jep.2004.03.006
Maes JM, Staines CL. 1991. Catálogo de los Chrysomelidae Coleoptera de Nicaragua. Revista Nicaraguense de Entomologia 18:1-53. http://www.bio-nica.info/RevNicaEntomo/RevNicaEntomo.htm

Maican S, Munteanu C. 2008. The diversity of urban coleopterans. In: Onete M, editor. Species monitoring in the central parks of Bucharest. Bucharest: Ars Docendi. p. 106-112.

Martins JKSS. 2015. Interações ecológicas associadas a Ipomoea Carnea subs. fistulosa (Martius e Choise) (Convolvulaceae) em uma área de Caatinga no Nordeste do Brasil [master's dissertation]. Recife (PE): Universidade Federal Rural de Pernambuco.

Oliveira PV, Ferreira JC, Moura FS, Lima GS, de Oliveira FM, Oliveira PES, Conserva LM, Giulietti AM, Lemos RPL. 2010. Larvicidal activity of 94 extracts from ten plant species of northeastern of Brazil against Aedes aegypti L. (Diptera: Culicidae). Parasitology Research 107:403-407. https://doi.org/10.1007/s00436-010-1880-4

Portman SL, Frank JH, McSorley R, Leppla NC. 2010. Nectar-seeking and host-seeking by Larra bicolor (Hymenoptera: Crabronidae), a parasitoid of Scapteriscus mole crickets (Orthoptera: Gryllotalpidae). Environmental Entomology 39(3):939-943. https://doi.org/10.1603/ en09268

Rafael JA, Limeira-de-Oliveira F, Hutchings RW, Miranda GFG, Silva Neto AM, Somavilla A, Camargo A, Asenjo A, Pinto ÂP, Bello AM, et al. 2020. Insect (Hexapoda) diversity in the oceanic archipelago of Fernando de Noronha, Brazil: updated taxonomic checklist and new records. Revista Brasileira de Entomologia 64(3):e20200052. https://doi.org/10.1590/1806-9665-rbent-2020-0052

Sassi D. 2018. Revision of the Metallactus kollari species-group with a new diagnosis of the genus (Coleoptera: Chrysomelidae: Cryptocephalinae). Zootaxa 4413(1):57-110. https://doi.org/10.11646/zootaxa.4413.1.2

\section{Agradecimientos / Acknowledgments:}

The authors are deeply grateful to Davide Sassi for confirming the genus of the beetle and pointing to a new species (not yet described). We thank Letícia Ribes de Lima for identifying the plant and the editor and reviewers for contributions that improve the manuscript.

Conflicto de intereses / Competing interests:

The authors declare no conflict of interest.

Rol de los autores / Authors Roles:

Duarte-de-Melo J: (1) Conceptualization; (2) Investigation; (3) Methodology; (4) Writing - original draft; (5) Writing - review and editing. Cajé, SOS: (1) Investigation; (2) Methodology; (3) Writing - original draft; (4) Writing - review and editing. Lima, IMM: (1) Project administration; (2) Supervision; (3) Writing review and editing.

Fuentes de financiamiento / Funding:

This research received no specific grant from any funding agency, commercial or not-for-profit sectors.

Aspectos éticos / legales; Ethics / legals:

Authors declare that they did not violate or omit ethical or legal norms in this research. 